\title{
THE FIXED-POINT-SPACE DIMENSION FUNCTION FOR A FINITE GROUP REPRESENTATION
}

\author{
I. M. ISAACS
}

(Communicated by Warren J. Wong)

\begin{abstract}
Given a complex representation of a finite group $G$, construct the integer valued function $\alpha$ on $G$ by setting $\alpha(g)$ to be the dimension of the fixed-point-space of $g$ in the module corresponding to the given representation. Usually, $\alpha$ is not a generalized character of $G$ and for trivial reasons $|G| \alpha$ is always a generalized character. The main result of this paper is that $e_{\alpha}$ is always a generalized character, where $e$ is the exponent of $G$.
\end{abstract}

\section{INTRODUCTION AND STATEMENT OF RESULTS}

Let $G$ be a finite group and let $\alpha$ be a $\mathrm{Z}$-valued function on $G$ which is constant on rational-classes, (i.e. $\alpha(x)=\alpha(y)$ if the cyclic groups $\langle x\rangle$ and $\langle y\rangle$ are $G$-conjugate.) Although $\alpha$ need not be a generalized character (i.e. a Z-linear combination of $\operatorname{Irr}(G)$ ), it is always true that $|G| \alpha$ is a generalized character. (This follows immediately from the observation that for each $\chi \in$ $\operatorname{Irr}(G)$, the sum of the values over each rational-class lies in $\mathbf{Z}$.)

For certain natural functions, $\alpha$, a multiplier $m$ smaller than $|G|$ is sufficient to make $m \alpha$ a generalized character. Our main result is that if $\alpha(x)$ denotes the dimension of the fixed-point space of $x$ in some $\mathbf{C}$-representation of $G$, then $e \alpha$ is a generalized character, where $e$ is the exponent of $G$.

If $\mathscr{X}$ is a $\mathbf{C}$-representation of $G$ affording the character $\chi$, then the fixedpoint-space dimension function $\alpha$ associated with $\mathscr{X}$ can be computed from $\chi$ by the formula $\alpha(x)=\left[\chi_{\langle x\rangle}, 1_{\langle x\rangle}\right]$, where $[\cdot, \cdot]$ denotes the character inner product. We introduce the notation $\hat{\chi}$ for this function.

Theorem 1. Let $\chi$ be any character of $G$. Then $e \hat{\chi}$ is a generalized character, where $e$ is the exponent of $G$ and $\hat{\chi}(x)=\left[\chi_{\langle x\rangle}, 1_{\langle x\rangle}\right]$ for $x \in G$.

In order to prove that $e \hat{\chi}$ is a generalized character of $G$, it suffices by Brauer's charaterization of characters to show that the restriction $(e \hat{\chi})_{N}$ is a generalized character of $N$ for every nilpotent subgroup $N \subseteq G$. Because

Received by the editors September 19, 1988.

1980 Mathematics Subject Classification (1985 Revision). Primary 20C15.

Research partially supported by a grant from the National Science Foundation. 
$(\hat{\chi})_{N}=\hat{\chi}_{N}$ and the exponent of $N$ divides that of $G$, it is enough to prove Theorem 1 in the case that $G$ is nilpotent.

We need to show (when $G$ is nilpotent) that $e[\hat{\chi}, \zeta] \in \mathbf{Z}$ for all irreducible characters $\zeta$ of $N$. In order to make our inductive proof of this work, we need to generalize it.

Proposition 2. Let $G$ be nilpotent and suppose $\chi$ is a character of $G$ and $\zeta$ is a generalized character. Then $m[\hat{\chi}, \zeta] \in \mathbf{Z}$, where $m$ is any positive integer such that $\zeta(x)=0$ whenever $x^{m} \neq 1$.

In particular, the condition that $\zeta(x)=0$ whenever $x^{m} \neq 1$ is vacuously satisfied if $m=e$, the exponent of $G$. Proposition 2, therefore, includes the nilpotent group case of Theorem 1 and so implies the general case by Brauer's theorem.

\section{ProOFS}

If $\chi$ is a complex-valued function on an arbitrary finite group $G$ and $n$ is a positive integer, we write $\chi^{(n)}$ to denote the function on $G$ defined by $\chi^{(n)}(x)=\chi\left(x^{n}\right)$. It is well known that if $\chi$ is a character (or a generalized character) of $G$, then $\chi^{(n)}$ is a generalized character. (See, for instance, [1, Problem 4.7].)

Somewhat analogously, we define $\chi^{(1 / n)}$ by the formula

$$
\chi^{(1 / n)}(x)=\sum_{\substack{y \in G \\ y^{n}=x}} \chi(y) .
$$

Lemma 3. If $\chi$ is a generalized character of $G$ and $n$ is a positive integer, then $\chi^{(1 / n)}$ is a generalized character.

Proof. Let $\zeta \in \operatorname{Irr}(G)$. Then

$$
\begin{aligned}
& {\left[\chi^{(1 / n)}, \zeta\right]=\frac{1}{|G|} \sum_{x \in G} \chi^{(1 / n)}(x) \overline{\zeta(x)}} \\
& =\frac{1}{|G|} \sum_{x \in G} \sum_{\substack{y \in G \\
y^{n}=x}} \chi(y) \overline{\zeta(x)} \\
& =\frac{1}{|G|} \sum_{y \in G} \chi(y) \overline{\zeta\left(y^{n}\right)} \\
& =\left[\chi, \zeta^{(n)}\right] \text {. }
\end{aligned}
$$

Since $\zeta^{(n)}$ is a generalized character, we have $\left[\chi, \zeta^{(n)}\right] \in \mathbf{Z}$ and it follows that $\chi^{(1 / n)}$ is a generalized character.

We need one further lemma for our proof of Proposition 2. 
Lemma 4. Let $N \triangleleft G$ with $G / N$ cyclic, and suppose $\chi$ is a generalized character of $G$ such that $\chi(x)=0$ for all $x \in G-N$. Then $\chi=\psi^{G}$ for some generalized character $\psi$ of $N$.

Proof. Let $C=\operatorname{Irr}(G / N)$ so that $C$ may be viewed as a group of linear characters of $G$ and $C$ acts on $\operatorname{Irr}(G)$ by multiplication. If $\lambda \in C$, then $\chi=\chi \lambda$ since $\chi(x)=0$ if $\lambda(x) \neq 1$. It follows that $\chi$ is a Z-linear combination of sums of orbits of the action of $C$ on $\operatorname{Irr}(G)$ and so it suffices to show that each orbit sum is induced from some character of $N$.

Let $\xi \in \operatorname{Irr}(G)$ and let $\eta$ be the sum of the $C$-orbit containing $\xi$. If $\alpha$ is any irreducible constituent of $\xi_{N}$, we will complete the proof by showing that $\alpha^{G}=\eta$.

If $x \in G-N$, we can choose $\lambda \in C$ with $\lambda(x) \neq 1$. Since $\eta$ is a $C$-orbit sum, we have $\eta \lambda=\eta$ and so $\eta(x)=0$. Also, $\alpha^{G}(x)=0$ and so $\eta$ and $\alpha^{G}$ agree on $G-N$.

Each irreducible constituent of $\eta$ has the form $\xi \mu$ for some $\mu \in C$. Since $\mu_{N}=1_{N}$, we have $(\xi \mu)_{N}=\xi_{N}$ and thus $\eta_{N}$ is a multiple of $\xi_{N}$ which is a multiple of the sum of the $G$-orbit of $\alpha$. Also, $\left(\alpha^{G}\right)_{N}$ is a multiple of the $G$-orbit sum of $\alpha$ and thus, to show that $\eta$ and $\alpha^{G}$ agree on $N$ (and thereby complete the proof), it suffices to compare degrees and show that $\eta(1)=\alpha^{G}(1)$.

Let $B \subseteq C$ be the stabilizer of $\xi$. Then $\eta(1)=|C: B| \xi(1)$. If $T \subseteq G$ is the stabilizer of $\alpha$, then $\xi=\beta^{G}$ where $\beta \in \operatorname{Irr}(T)$ and $\beta_{N}$ is a multiple of $\alpha$. Since $T / N$ is cyclic, we have $\beta_{N}=\alpha$ and so $\xi(1)=|G: T| \alpha(1)$ and $\eta(1)=$ $|C: B \| G: T| \alpha(1)$. Of course, $\alpha^{G}(1)=|G: N| \alpha(1)$ and so we must show that $|G: N|=|G: T||C: B|$. It suffices, therefore, to prove that $|T: N|=|C: B|$.

We claim that $B=\operatorname{Irr}(G / T)$. This would give $|C: B|=|C| /|B|=|G: N| /$ $|G: T|=|T: N|$, as required. To prove the claim, let $\mu \in B$. Then

$$
\xi=\xi \mu=\beta^{G} \mu=\left(\beta \mu_{T}\right)^{G}
$$

and $\left(\beta \mu_{T}\right)_{N}=\beta_{N}=\alpha$. However, $\beta$ is the unique character of $T$ lying over $\alpha$ which induces $\xi$, and thus $\beta \mu_{T}=\beta$. Since $\beta_{N}$ is irreducible and $N \subseteq \operatorname{ker}\left(\mu_{T}\right)$, it follows that $\mu_{T}=1_{T}$. (Use [1, 6.17], for instance). Thus $\mu \in \operatorname{Irr}(G / T)$ as required.

Conversely, suppose $\mu \in \operatorname{Irr}(G / T)$. Then

$$
\xi \mu=\beta^{G} \mu=\left(\beta \mu_{T}\right)^{G}=\beta^{G}=\xi
$$

and so $\mu \in B$. The proof is now complete.

Proof of Proposition 2. The map $\chi \mapsto \hat{\chi}$ is additive and so it is no loss to assume that $\chi$ is an irreducible character. We proceed by induction on $\chi(1)$.

First, suppose $\chi(1)=1$ so that $\chi$ is linear. Writing $K=\operatorname{ker}(\chi)$, we see that $\hat{\chi}(x)=0$ if $x \notin K$ and $\hat{\chi}(x)=1$ if $x \in K$. Let $N=\left\{g \in G \mid g^{m} \in K\right\}$, the preimage in $G$ of the set of elements in $G / K$ with order dividing $m$. Since $G / K$ is cyclic, it follows that $N$ is a subgroup and $|N / K|$ divides $m$. Thus 
$m \hat{\chi}_{N}$ is a multiple of $|N / K| \hat{\chi}_{N}=\left(1_{K}\right)^{N}$. In particular, $m \hat{\chi}_{N}$ is a character of $N$.

If $x \in G-N$, then $x^{m} \notin K$ and so $x^{m} \neq 1$ and $\zeta(x)=0$ by hypothesis. Since $G / N$ is cyclic, Lemma 4 tells us that $\zeta=\eta^{G}$ some generalized character $\eta$ of $N$. Then $m[\hat{\chi}, \zeta]=\left[m \hat{\chi}, \eta^{G}\right]=\left[m \hat{\chi}_{N}, \eta\right]$ and this lies in $\mathbf{Z}$ since $m \hat{\chi}_{N}$ is a character.

Now assume $\chi(1)>1$. The nilpotence of $G$ guarantees that we can write $\chi=\psi^{G}$, where $\psi \in \operatorname{Irr}(H)$ and $H \triangleleft G$ has prime index $p$. Then $\chi_{H}=$ $\psi_{1}+\psi_{2}+\cdots+\psi_{p}$ where $\psi_{1}=\psi$ and the $\psi_{i}$ constitute an orbit of the action of $G$ on $\operatorname{Irr}(H)$. Thus $\hat{\chi}_{H}=\sum_{i} \hat{\psi}_{i}$ and the $\hat{\psi}_{i}$ are all $G$-conjugate. We will also need to be able to compute $\hat{\chi}(x)$ if $x \in G-H$; we work with modules to do this.

Let $V$ be a $\mathbf{C} G$-module affording $\chi$. Then $V=V_{1} \dot{+} V_{2} \dot{+} \cdots \dot{+} V_{p}$, where the $V_{i}$ are $\mathbf{C H}$-submodules of $V$ affording the characters $\psi_{i} \in \operatorname{Irr}(H)$ and these submodules are permuted by $G$. In fact, if $x \in G-H$, then $\langle x\rangle$ permutes the $V_{i}$ transitively and we may assume $x$ carries $V_{i}$ to $V_{i+1}$ for $1 \leq i<p$ and $x$ carries $V_{p}$ to $V_{1}$.

Holding $x \in G-H$ fixed, we can define a map $w \mapsto \tilde{w}$ from $V_{1}$ into $V$ by setting $\tilde{w}=w+w x+w x^{2}+\cdots+w x^{p-1}$. This is a $\mathbf{C}$-linear transformation which is injective since $\tilde{w} \neq 0$ when $w \neq 0$ because $w x^{i} \in V_{i+1}$ for $0 \leq i \leq$ $p-1$ and the sum $\sum V_{i}$ is direct. If $w$ is a fixed point of $x^{p}$ in $V_{1}$, then clearly $\tilde{w}$ is fixed by $x$. Conversely, if $v \in V$ is a fixed point of $x$, then writing $v=v_{1}+v_{2}+\cdots+v_{p}$ with $v_{i} \in V_{i}$ and comparing the projections of the equal vectors $v$ and $v x$ into the $V_{i}$, we see that $v_{1} x=v_{2}, v_{2} x=$ $v_{3}, \ldots, v_{p-1} x=v_{p}$ and $v_{p} x=v_{1}$. It follows that $v=\tilde{v}_{1}$ and that $v_{1}$ is fixed by $x^{p}$.

We have proved that the map $w \mapsto \tilde{w}$ defines an isomorphism of the fixedpoint space of $x^{p}$ in $V_{1}$ onto the fixed-point space of $x$ in $V$. This shows that $\hat{\chi}(x)=\hat{\psi}\left(x^{p}\right)$ for all $x \in G-H$.

Now

$$
m[\hat{\chi}, \zeta]=\frac{m}{|G|} \sum_{x \in G} \hat{\chi}(x) \overline{\zeta(x)}=\frac{m}{|G|} S_{1}+\frac{m}{|G|} S_{2}
$$

where $S_{1}$ is the sum for $x \in H$ and $S_{2}$ is the sum for $x \in G-H$. When $x \in H$, we have $\hat{\chi}(x)=\sum_{i} \hat{\psi}_{i}(x)$ and so

$$
S_{1}=|H|\left[\left(\sum_{i} \hat{\psi}_{i}\right), \zeta_{H}\right]=p|H|\left[\hat{\psi}, \zeta_{H}\right]
$$

since all $\left[\hat{\psi}, \zeta_{H}\right]$ are equal. (This is because the $\hat{\psi}_{i}$ are $G$-conjugate). Thus $(m /|G|) S_{1}=m\left[\hat{\psi}, \zeta_{H}\right]$ and this lies in $\mathbf{Z}$ by the inductive hypothesis since $\psi(1)<\chi(1)$. 
To evaluate $S_{2}$, define $\xi(y)=\sum_{x \in G-H, x^{p}=y} \zeta(x)$. Then

$$
\begin{aligned}
S_{2} & =\sum_{x \in G-H} \hat{\chi}(x) \overline{\zeta(x)} \\
& =\sum_{x \in G-H} \hat{\psi}\left(x^{p}\right) \overline{\zeta(x)} \\
& =\sum_{y \in H} \hat{\psi}(y) \overline{\xi(y)} \\
& =|H|[\hat{\psi}, \xi]
\end{aligned}
$$

and so $(m /|G|) S_{2}=(m / p)[\hat{\psi}, \xi]$ and we need to show that this lies in $\mathbf{Z}$.

If $x \in G-H$ and $x^{p}=y \in H$, then $p$ divides the order of $x$. If $p \nmid m$, then $x^{m} \neq 1$ and so $\zeta(x)=0$ by hypothesis. It follows that $\xi$ is identically zero if $p \nmid m$ and there is nothing to prove in this case. Suppose, therefore, that $p \mid m$. If $x^{m}=1$ and $x^{p}=y$, then $y^{(m / p)}=1$. Therefore, if $y^{(m / p)} \neq 1$, we have $\zeta(x)=0$ for all $x$ with $x^{p}=y$ and so $\xi(y)=0$.

Now

$$
\xi(y)=\sum_{\substack{x \in G \\ x^{p}=y}} \zeta(x)-\sum_{\substack{x \in H \\ x^{p}=y}} \zeta(x)
$$

and so. $\xi=\left(\zeta^{(1 / p)}\right)_{H}-\left(\zeta_{H}\right)^{(1 / p)}$ and this is a generalzied character by Lemma 3. Since $\xi(y)=0$ if $y^{(m / p)} \neq 1$, the inductive hypothesis yields $(m / p)[\hat{\psi}, \xi] \in \mathbf{Z}$, as required.

The proof of our main result, Theorem 1, is now complete.

\section{FURTHER REMARKS}

If we drop the nilpotence hypothesis from Proposition 2, we get a statement properly stornger than Theorem 1 . Is this statement true?

Theorem 5. Let $\chi$ be a character of an arbitrary finite group $G$ and let $\zeta$ be a generalized character of $G$ with the property that $\zeta(x)=0$ whenever $x^{m} \neq 1$ for some fixed positive integer $m$. Then $m[\hat{\chi}, \zeta] \in \mathbf{Z}$.

Proof. By Brauer's theorem on induced characters, the principal character $1_{G}$ can be written as a sum of induced generalized characters $\alpha_{i}^{G}$ where the $\alpha_{i}$ are characters of certain nilpotent subgroups $N_{i} \subseteq G$. Then

$$
\zeta=\zeta 1_{G}=\sum_{i} \zeta\left(\alpha_{i}\right)^{G}=\sum_{i}\left(\zeta_{N_{i}} \alpha_{i}\right)^{G} .
$$

Thus

$$
m[\hat{\chi}, \zeta]=\sum m\left[\hat{\chi},\left(\zeta_{N_{i}} \alpha_{i}\right)^{G}\right]=\sum_{i} m\left[\hat{\chi}_{N_{i}}, \zeta_{N_{i}} \alpha_{i}\right]
$$

and this is a sum of integers by Proposition 2, since the $\zeta_{N_{i}} \alpha_{i}$ are generalized characters which vanish on elements $x \in N_{i}$ such that $x^{m} \neq 1$. 
We began this paper with the observation that if $\alpha$ is any $\mathbf{Z}$-valued function on $G$, constant on rational classes, then $|G| \alpha$ is a generalized character; equivalently $|G|[\alpha, \zeta] \in \mathbf{Z}$ for each $\zeta \in \operatorname{Irr}(G)$. The point of Theorem 1 is that in certain situations, for particular $\alpha$, the coefficient $|G|$ can be reduced uniformly; i.e. for all $\zeta$. It is true (and quite trivial) that for particular $\zeta$ we can reduce the coefficient for all $\alpha$. We conclude with this observation.

Theorem 6. Let $\alpha$ be $\mathbf{Z}$-valued and constant on rational-classes of $G$. Then

$$
\frac{|G|}{\zeta(1)}[\alpha, \zeta] \in \mathbf{Z}
$$

for all $\zeta \in \operatorname{Irr}(G)$.

Proof. Let $x_{1}, x_{2}, \ldots, x_{k}$ be representatives for the conjugacy classes $K_{1}, \ldots$, $K_{k}$ of $G$. Then

$$
\begin{aligned}
\frac{|G|}{\zeta(1)}[\alpha, \zeta] & =\sum_{x \in G} \alpha(x) \frac{\overline{\zeta(x)}}{\zeta(1)} \\
& =\sum_{i=1}^{k} \alpha(x) \frac{\overline{\zeta\left(x_{i}\right)}}{\zeta(1)}\left|K_{i}\right|
\end{aligned}
$$

and this is an algebraic integer since $\zeta\left(x_{i}\right)\left|K_{i}\right| / \zeta(1)$ is integral. Also, $(|G| / \zeta(1))[\alpha, \zeta]$ is rational since $|G|[\alpha, \zeta] \in \mathbf{Z}$. The result follows.

In view of Theorems 1 and 6 , one might guess that if $\chi, \zeta \in \operatorname{Irr}(G)$, then $(e / \zeta(1))[\hat{\chi}, \zeta] \in \mathbf{Z}$, where $e$ is the exponent of $G$. This is false since if $G$ has order $p^{3}$ and exponent $p$ (where $p$ is prime), then $[\hat{\chi}, \chi]=1 / p$ for $\chi \in \operatorname{Irr}(G)$ with $\chi(1)=p$.

\section{REFERENCES}

1. I. M. Isaacs, Character theory of finite groups, Academic Press, New York, 1976.

Department of Mathematics, University of Wisconsin, Madison, Wisconsin 53706 